\title{
Prognostic Impact and Clinicopathological Features of Multiple Colorectal Cancers and Extracolorectal Malignancies: A Nationwide Retrospective Study
}

\author{
Kentaro Ochiai ${ }^{a}$ Kazushige Kawai ${ }^{a}$ Hiroaki Nozawa ${ }^{a}$ Kazuhito Sasaki ${ }^{a}$ \\ Manabu Kaneko ${ }^{a}$ Koji Murono ${ }^{a}$ Shigenobu Emoto ${ }^{a}$ Hiroaki Ishii $^{a}$ \\ Hirofumi Sonoda $^{a}$ Shinichi Yamauchi ${ }^{b}$ Kenichi Sugiharab Soichiro Ishihara ${ }^{b}$ \\ aDepartment of Surgical Oncology, Faculty of Medicine, The University of Tokyo, Tokyo, Japan; bepartment of \\ Surgical Oncology, Tokyo Medical and Dental University, Tokyo, Japan
}

\section{Keywords}

Multiple primary malignancies - Multiple cancers .

Colorectal cancer · Prognosis

\begin{abstract}
Introduction: Multiple primary malignancies (MPMs) are likely to develop in patients with colorectal cancer (CRC); however, their prognoses are unclear. This study aims to investigate the prognostic impacts and clinicopathological features of multiple CRCs and extracolorectal malignancies (EMs) with CRC. Methods: We retrospectively evaluated a total of 22,628 patients with stage I-III CRC who underwent curative resection at 24 referral institutes in Japan between January 2004 and December 2012. MPMs were classified as synchronous CRCs (SCRCs), metachronous CRCs, synchronous EMs (SEMs), and metachronous EMs. Results: The presence of SCRCs (odds ratio 1.54, $p<0.001$ ) was independently associated with SEMs in the multivariate analyses. SEMs were the strongest poor prognostic factor for OS (hazard ratio [HR] 2.21, $p<0.001)$ and RFS (HR 1.69, $p<0.001)$ compared with age, sex, and primary $\mathrm{T}$ and $\mathrm{N}$ factors. The inci-
\end{abstract}

karger@karger.com www.karger.com/dig

Karger $\stackrel{\text { ' }}{5}$

BOPEN ACCESS
(C) 2021 The Author(s)

Published by S. Karger AG, Basel

This is an Open Access article licensed under the Creative Commons Attribution-NonCommercial-4.0 International License (CC BY-NC) (http://www.karger.com/Services/OpenAccessLicense), applicable to the online version of the article only. Usage and distribution for commercial purposes requires written permission. dence of stomach cancer was the highest in EMs, followed by lung, breast, and prostate cancers. Multiple CRCs were evenly distributed throughout the right-side colon to the rectum. Discussion/Conclusion: SEMs were a strong poor prognostic factor for patients with stage I-III CRC. Patients with CRC, particularly those with SCRCs, should be surveyed for SEMs, especially for stomach and lung cancers.

(c) 2021 The Author(s).

Published by S. Karger AG, Basel

\section{Introduction}

Colorectal cancer (CRC) is the third most common malignant disease worldwide and the second most common cause of cancer mortality [1]. Patients with CRC are likely to develop multiple primary malignancies (MPMs). In fact, patients with CRC have a 1.4-1.7-fold greater risk of developing a second CRC compared with the healthy population $[2,3]$. CRC is also likely accompanied by extracolorectal malignancies (EMs). Several hereditary diseases, such as Lynch syndrome or familial adenomatous polyposis, increase the risk of many types of cancers, in- 
cluding CRC. However, patients with CRC without these hereditary diseases are also at a high risk for accompaniment by various types of primary malignancies in extracolorectal organs [4]. The popularization of health screening and the progression of technology for diagnosis and treatment have enabled early detection and appropriate treatment of CRC. Therefore, the number of CRC survivors has increased in recent years. According to the multiinstitutional registry of the Japanese Society for Cancer of the Colon and Rectum (JSCCR), the 5-year survival of all Japanese patients with CRC was $72 \%$, which increased to $82 \%$ when focusing on patients with stage 0 -III CRC who underwent curative resection [5]. Therefore, it is important to consider the effects of MPMs when deciding on a treatment strategy or postoperative surveillance for patients with CRC.

Although several studies have reported about MPMs in patients with CRC, the results have greatly differed owing to differences in the study designs and cohorts $[6,7]$. Moreover, the prognostic impact of EMs and multiple CRCs has not been completely investigated. There are few reports regarding the prognoses of patients with CRC with EMs. We previously reported that EMs, particularly synchronous EMs (SEMs), are strongly associated with poor survival [8]; however, whether this result can be generalized remains uncertain because it was a single-center study. Therefore, validation in a Japanese nationwide cohort is necessary. Several studies have reported that multiple CRCs are associated with poor prognoses $[9,10]$, whereas other studies have reported no difference in prognoses $[11,12]$. Therefore, associating multiple CRCs with prognoses remains controversial [13]. According to our previous report [12], patients with synchronous CRCs (SCRCs) had poorer relapse-free survival (RFS) than those with solitary CRCs; in contrast, the presence of SCRCs did not significantly affect overall survival (OS). However, this was a single-center study, the study population was relatively small, and it contained many patients with stage IV SCRSs. Thus, it could not examine the prognostic impact of multiple CRCs.

In turn, OS or RFS has been used for prognostic analyses in many reported clinical studies. The events in these indexes not only include CRC-related deaths but also any other causes of death, including MPM-related deaths [14, 15]. Therefore, it is important to consider the effects of MPMs when evaluating OS or RFS. Unfortunately, the definitions of these endpoints are frequently missing in many studies [14]. This study aimed to investigate the prognostic impacts and clinicopathological features of multiple CRCs and EMs with CRC.

\section{Materials and Methods}

\section{Study Population}

We retrospectively collected the data from 24 referral hospitals in Japan (see the Acknowledgment section). The study population consisted of 22,628 consecutive patients with pathological stage I-III CRC who had undergone curative resection between January 2004 and December 2012 in each institute. The following patients were excluded from this study: 249 patients who were treated for reasons other than carcinoma, 30 patients with hereditary colorectal disease, such as Lynch syndrome or familial adenomatous polyposis, $91 \mathrm{pa}-$ tients with inflammatory bowel diseases, and 371 patients with inadequate clinicopathological data. Thus, 21,887 patients were included in the analysis (Fig. 1). The onset of multiple CRCs and EMs was defined according to the Japanese Classification of Colorectal, Appendiceal, and Anal carcinoma, 7th edition [16]; multiple CRCs and EMs diagnosed within a year before and after surgery were defined as SCRCs and SEMs. Those diagnosed at $>1$ year before and after surgery were defined as metachronous CRCs (MCRCs) and metachronous EMs (MEMs). Patients were surveyed according to the JSCCR guidelines [17] for treatment of CRC at least 5 years after surgery by physical examination, tumor marker (carcinoembryonic antigen and carbohydrate antigen 19-9) measurements, CT examination, and colonoscopy. Typically, tumor markers were examined every 3 months, CT was performed every 6 months, and colonoscopy was performed every year. The median follow-up period was 70 months. OS was defined as death for any reason (event cases) or loss to follow-up (censored cases). RFS was defined as recurrence of CRC or death for any reason (event cases) or loss to follow-up (censored cases) as recommended by Punt et al. [14].

The Ethics Committee of the University of Tokyo (No. 2018028NI-[1]) and local ethics committees in each participant institute approved the study protocol. The study was conducted in accordance with the Declaration of Helsinki. Informed consent was replaced by the obligation of information to the participants and the right of participants to opt out due to the study's retrospective nature.

\section{Statistical Analyses}

All analyses were performed using JMP Pro 14.2 (SAS Institute Inc., Cary, NC, USA, www.jmp.com). The associations between the clinicopathological background and the presence of SCRCs or SEMs were evaluated using Pearson's $\chi^{2}$ test as the univariate analysis and logistic regression models as the multivariate analysis. $\mathrm{Cu}$ mulative survival was estimated using the Kaplan-Meier method and compared using the log-rank test. Hazard ratios (HRs) for OS and RFS were calculated using univariate and multivariate Cox proportional hazards regression models. All reported $p$ values are 2 -tailed, and $p$ values of $<0.05$ were considered significant.

\section{Results}

\section{Characteristics}

The patient flow and incidences of multiple CRCs and EMs are shown in Figure 1. Of the 21,887 patients, 1,884 (8.6\%) had multiple CRCs, including 1,221 (5.6\%) with SCRCs, and 3,444 (15.7\%) had EMs, including 859 (3.9\%) 
Fig. 1. Patient flow and incidence of multiple CRCs and EMs. CRCs, colorectal cancers; EMs, extracolorectal malignancies; SCRCs, synchronous CRCs; MCRCs, metachronous CRCs; SEMs, synchronous EMs; MEMs, metachronous EMs.

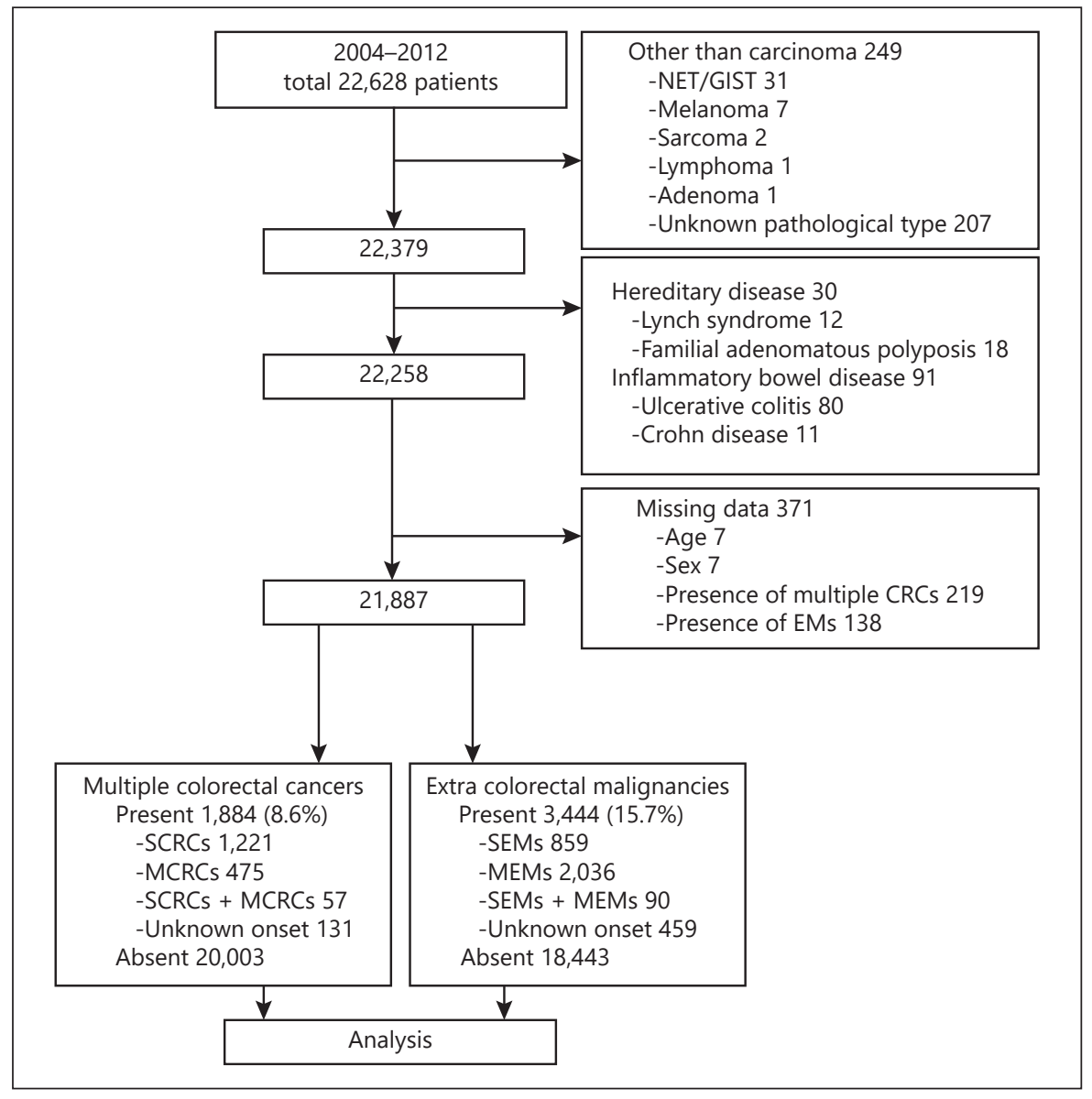

with SEMs. We first analyzed SCRCs and SEMs. As shown in Table 1, patients with SCRCs and SEMs were older ( $p$ $<0.001)$, more likely to be male $(p<0.001)$, and more commonly had the colon as the primary CRC site rather than the rectum (SCRCs: $p<0.001$; SEMs: $p=0.036$ ). We also evaluated the association between the presence of SCRCs and SEMs. SEMs were more frequent in patients with SCRCs than in those without SCRCs $(p<0.001)$; similarly, SCRCs were more frequent in patients with SEMs than in those without SEMs $(p<0.001)$.

\section{Risk Factors of SEMs}

In the logistic regression analysis, independent factors associated with SEMs included male sex (odds ratio [OR] $1.61, p<0.001$ ), older age (over 70) (OR 1.58, $p<0.001$ ), and presence of SCRCs (OR 1.54, $p<0.001)$ (Table 2).

\section{Survival Analyses}

The results of the prognostic analyses are shown in Figure 2 and Tables 3 and 4. As shown by the KaplanMeier curves (Fig. 2), patients with SEMs had poorer

Prognostic Impact of Multiple Primary Malignancies with CRC prognoses in OS $(p<0.001)$ and RFS $(p<0.001)$. Patients with SCRCs also had a poorer prognosis in OS $(p=0.049)$. Figure 3 shows Kaplan-Meier curves of CRC-related and EM-related death. The 5-year overall, CRC-related, and EM-related death rates were $13.5,7.1$, and $2.3 \%$, respectively. In the multivariate analyses (Tables 3,4), SEMs were an independent risk factor of poor prognosis in OS (HR 2.21, $p<0.001$ ) and RFS (HR 1.69, $p<0.001$ ). Notably, the impact of SEMs on OS was the strongest factor compared to the others. Conversely, there were no significant differences in OS and RFS with or without SCRCs in the multivariate analyses. A multivariate analysis of OS including the site of SEM is shown in online suppl. Table (see www.karger.com/doi/10.1159/000517271 for all online suppl. material). Among SEMs, stomach and lung cancers were independent risk factors for OS, while breast and prostate cancers were not.

\section{Distribution of EMs}

The details of EMs, which combined SEMs and MEMs, are shown in Table 5. Some of the 3,444 patients with EMs 
Table 1. Patients' characteristics according to the presence of EMs and multiple CRCs

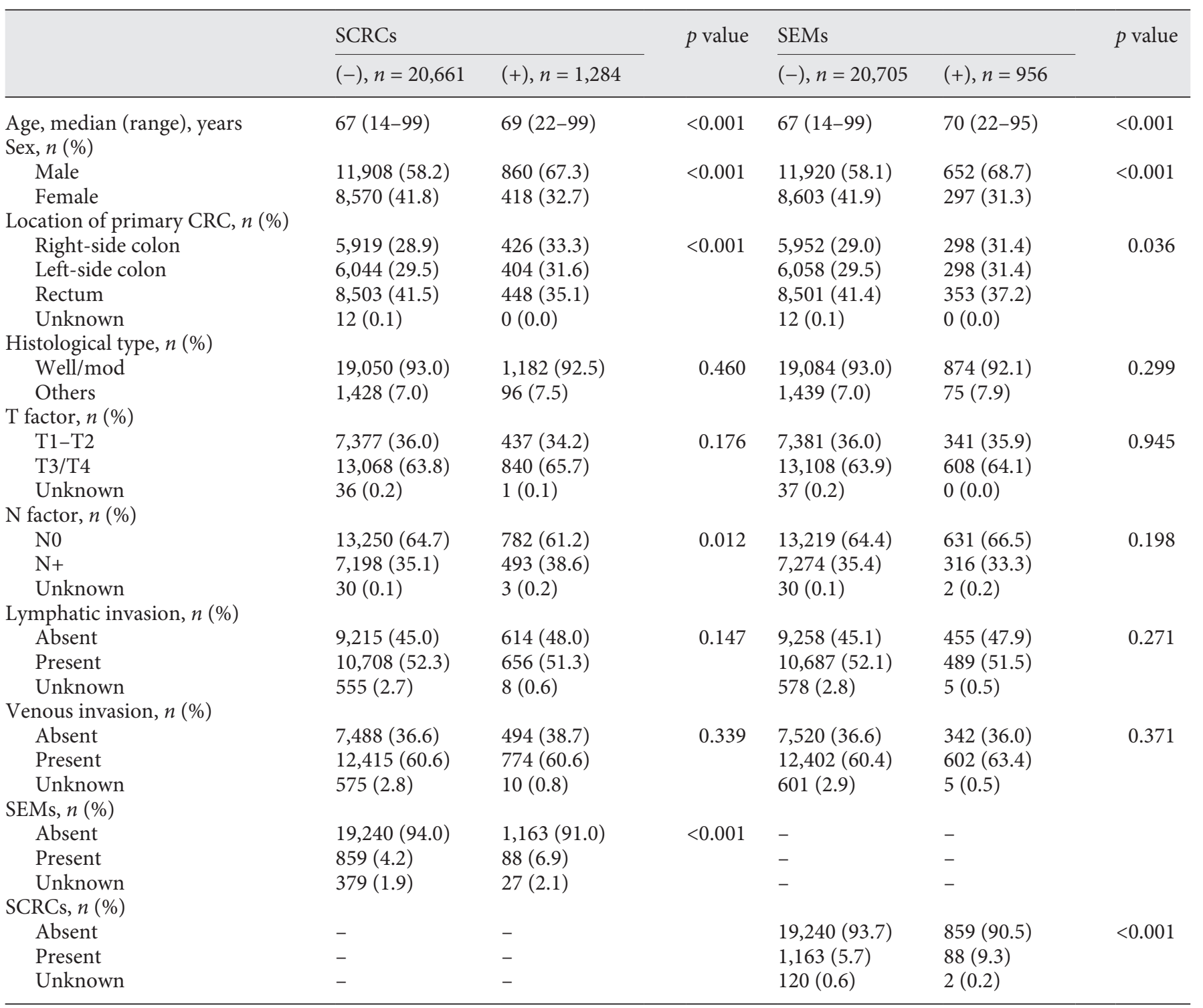

CRCs, colorectal cancers; EMs, extracolorectal malignancies; SCRCs, synchronous CRCs; SEMs, synchronous EMs.

had multiple types of malignancies; therefore, there were 3,752 EMs in total. Stomach cancer was the most frequent in males, followed by prostate and lung cancers. Breast cancer was the most common EM in females, followed by uterus/ovary, stomach, and lung cancers.

\section{Locations of Multiple CRCs}

The combinations of the sites of multiple CRCs are presented in Figure 4. CRCs were distributed evenly throughout the right side of the colon to the rectum. However, relatively few patients had cancer only in the rectal area.

\section{Discussion}

MPMs are defined as 2 or more malignancies that independently develop in a patient. The criteria were initially established by Warren and Gate [18] and are still widely used: (1) each tumor must present a definite picture of a malignancy, (2) each tumor must be distinct, and (3) the probability of one being a metastasis of the other must be excluded. Since the metachronous MPMs in our database had a fairly wide range of onset and the information about these lesions was insufficient, we focused on 
Table 2. Logistic regression analyses for the risk of SEMs

\begin{tabular}{lrrrrr}
\hline & \multirow{2}{*}{$\begin{array}{l}\text { Univariate } \\
p \text { value }\end{array}$} & \multicolumn{4}{l}{ Multivariate } \\
\cline { 4 - 6 } & & & $p$ value & OR & $95 \%$ CI \\
\hline Age (<70 vs. $\geq 70$ years) & $<0.001$ & $<0.001$ & 1.58 & $1.39-1.81$ \\
Sex (female vs. male) & $<0.001$ & $<0.001$ & 1.61 & $1.40-1.86$ \\
Primary tumor location (colon vs. rectum) & 0.010 & 0.060 & 0.88 & $0.76-1.01$ \\
SCRCs (absent vs. present) & $<0.001$ & $<0.001$ & 1.54 & $1.23-1.94$ \\
\hline
\end{tabular}

SEMS, synchronous extracolorectal malignancies; SCRCs, synchronous colorectal cancers.

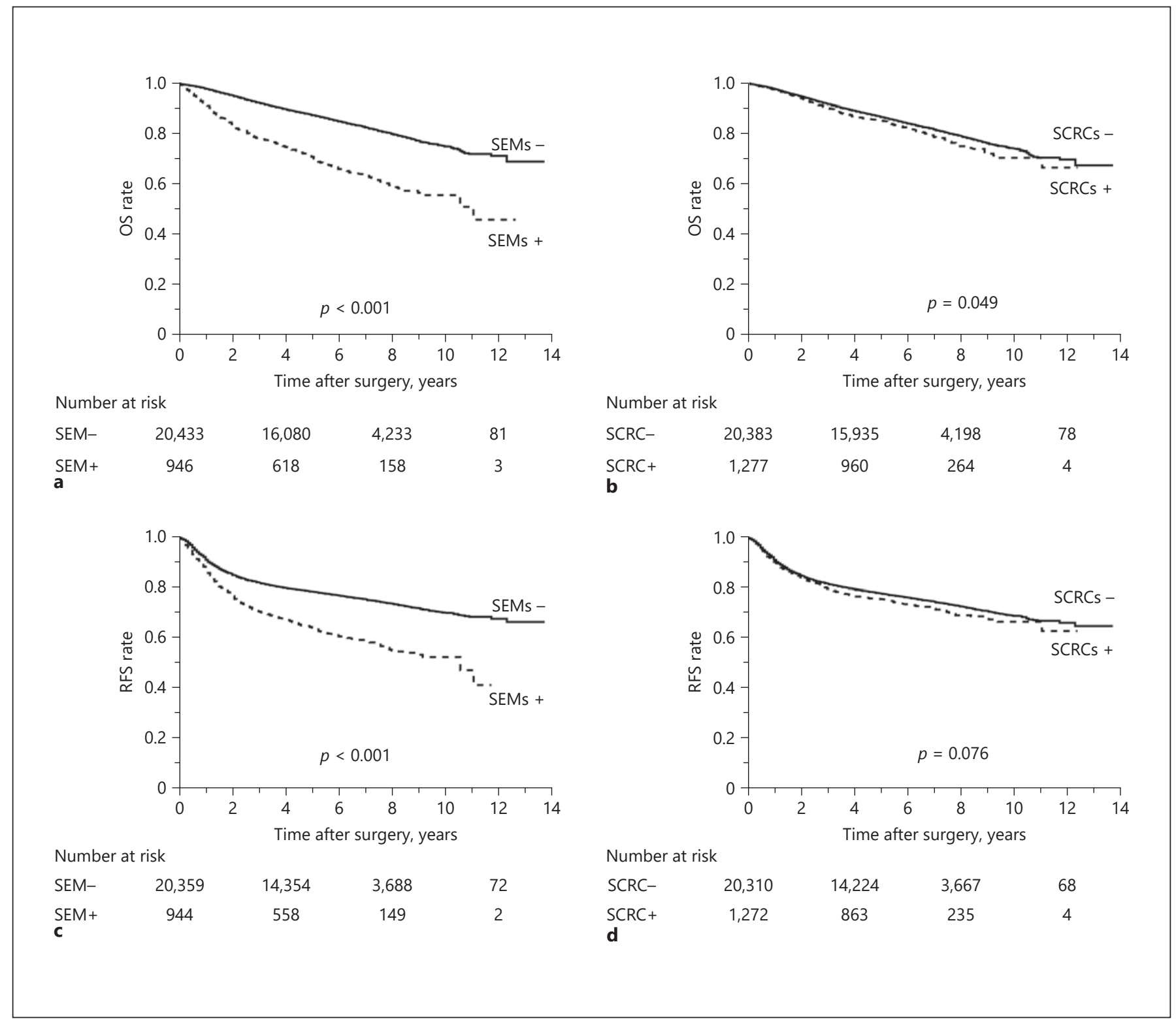

Fig. 2. Kaplan-Meier curves of OS (a, b) and RFS (c, d) according to the presence of EMs and multiple CRCs. OS, overall survival; RFS, relapse-free survival; CRCs, colorectal cancers; EMs, extracolorectal malignancies; SEMs, synchronous EMs; SCRCs, synchronous CRCs. 
Table 3. Cox proportional hazard models of OS

\begin{tabular}{|c|c|c|c|c|}
\hline & \multirow{2}{*}{$\begin{array}{l}\text { Univariate } \\
p \text { value }\end{array}$} & \multicolumn{3}{|c|}{ Multivariate } \\
\hline & & $p$ value & HR & $95 \% \mathrm{CI}$ \\
\hline Age ( $<70$ vs. $\geq 70$ years) & $<0.001$ & $<0.001$ & 2.02 & $1.88-2.16$ \\
\hline Sex (female vs. male) & $<0.001$ & $<0.001$ & 1.36 & $1.27-1.46$ \\
\hline Primary tumor location (colon vs. rectum) & $<0.001$ & $<0.001$ & 1.21 & $1.12-1.29$ \\
\hline SCRCs (absent vs. present) & 0.024 & 0.590 & 1.04 & $0.91-1.19$ \\
\hline SEMs (absent vs. present) & $<0.001$ & $<0.001$ & 2.21 & $1.96-2.48$ \\
\hline Histological type (well/mod vs. others) & $<0.001$ & $<0.001$ & 1.57 & $1.41-1.76$ \\
\hline $\mathrm{T}$ factor (T1-T2 vs. T3-T4) & $<0.001$ & $<0.001$ & 1.89 & $1.73-2.07$ \\
\hline pN factor (absent vs. present) & $<0.001$ & $<0.001$ & 2.03 & $1.86-2.21$ \\
\hline Lymphatic invasion (absent vs. present) & $<0.001$ & $<0.001$ & 1.32 & $1.22-1.43$ \\
\hline Venous invasion (absent vs. present) & $<0.001$ & $<0.001$ & 1.27 & $1.17-1.38$ \\
\hline Adjuvant chemotherapy (absent vs. present) & $<0.001$ & $<0.001$ & 0.61 & $0.56-0.66$ \\
\hline
\end{tabular}

OS, overall survival; SEMs, synchronous extracolorectal malignancies; SCRCs, synchronous colorectal cancers.

Table 4. Cox proportional hazard models of RFS

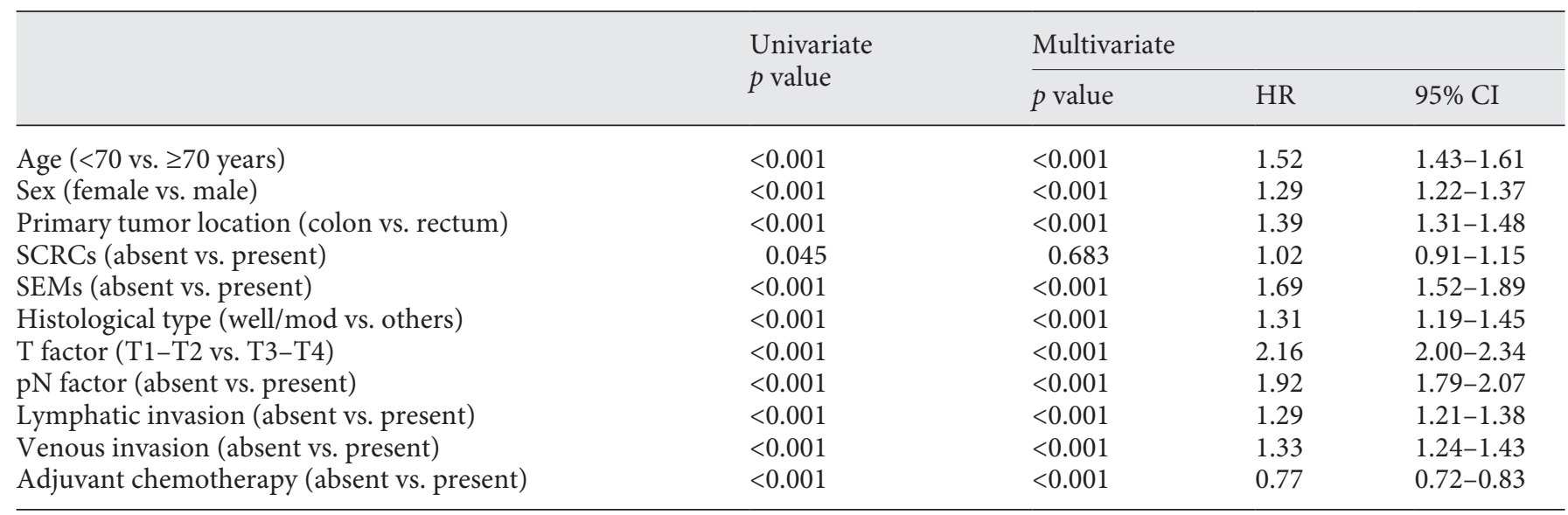

RFS, relapse-free survival; SEMs, synchronous extracolorectal malignancies; SCRCs, synchronous colorectal cancers.

synchronous MPMs, for which more reliable information was available, in the prognostic analysis. We examined the distribution of MPMs combined with metachronous and synchronous MPMs to provide suggestions for preoperative screening and postoperative surveillance.

Multivariate analyses revealed that older age (OR 1.58), male sex (OR 1.61), and the presence of SCRCs (OR 1.54) were associated with SEMs. Notably, the presence of SCRCs was thought to be an important independent factor for SEMs, comparable to age and sex. We can provide some reasonable explanations for these results. One possible explanation is genetic background. While we excluded the patients with a hereditary colorectal disease, there can be potential effects of genetic abnormalities. Microsatellite instability (MSI) is broadly known to be a risk factor not only for CRC but also for various types of malignancies, including cancers of the stomach, small intestine, and ovaries and transitional cell carcinoma of the upper urological tract [19]. Another possible reason is that both SCRCs and SEMs have common risk factors, such as smoking and drinking habits, environmental exposure, and comorbidities such as obesity and diabetes. Because these are risks for not only CRC but also various other malignancies [20], patients with SCRCs and SEMs sharing such risk factors is possible. In any case, in this large, multi-institutional study, patients with SCRCs had 
Table 5. Incidence of EMs by sites

\begin{tabular}{llll}
\hline \multirow{2}{*}{ Type of EMs } & \multicolumn{2}{l}{ Sex } & Total (\%) \\
\cline { 2 - 3 } & male (\%), $n=12,857$ & female (\%), $n=9,030$ & $906(4.1)$ \\
\hline Stomach & $683(5.3)$ & $223(2.5)$ & $515(2.4)$ \\
Lung & $351(2.7)$ & $164(1.8)$ & $367(1.7)$ \\
Breast & $17(0.1)$ & $350(3.9)$ & $350(1.6)$ \\
Prostate & $350(2.7)$ & - & $258(1.2)$ \\
Uterus/ovary & - & $258(2.9)$ & $326(1.5)$ \\
Hepatobiliary/pancreas & $227(1.8)$ & $99(1.1)$ & $178(0.8)$ \\
Esophagus & $160(1.2)$ & $18(0.2)$ & $175(0.8)$ \\
Head and neck & $121(0.9)$ & $54(0.6)$ & $159(0.7)$ \\
Kidney & $112(0.9)$ & $47(0.5)$ & $156(0.7)$ \\
Urinary tract & $125(1.0)$ & $31(0.3)$ & $125(0.6)$ \\
Hematopoietic/lymphoid & $81(0.6)$ & $10(0.5)$ & $40(0.2)$ \\
Skin & $30(0.2)$ & $5(0.1)$ & $20(0.1)$ \\
Brain/nerve & $15(0.1)$ & $9(0.1)$ & $19(0.1)$ \\
Bone/soft tissue & $10(0.1)$ & $5(0.1)$ & $20(0.1)$ \\
Unknown & $15(0.1)$ & $47(0.5)$ & $138(0.6)$ \\
Others & $91(0.7)$ & $1,364(15.1)$ & $3,752(17.1)$ \\
\hline Total & $2,388(18.6)$ & &
\end{tabular}

EMs, extracolorectal malignancies.
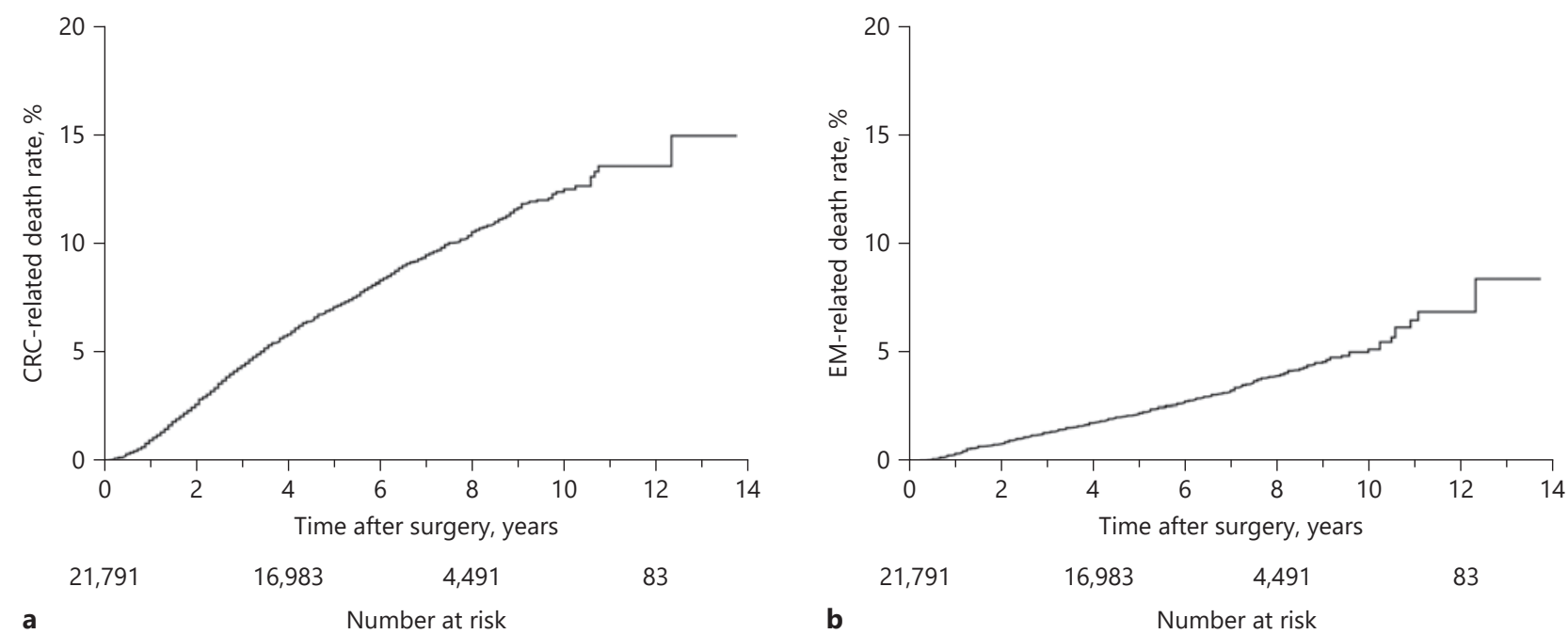

Fig. 3. Accumulative incidences of cancer-related death. Kaplan-Meier curves of accumulative incidences of death due to CRC (a) and EM (b). CRC, colorectal cancer; EM, extracolorectal malignancy.

a 1.54 times greater risk of SEMs compared to those without SCRCs. Thus, patients with SCRCs should be surveyed for SEMs with special care. Specifically, as described later, stomach cancer and lung cancer were particularly common and associated with worse prognoses; thus, gas- troscopy (GS) and chest CT (not only for screening for lung metastases from CRC but also for screening for primary lung cancer) should be considered for these patients. 


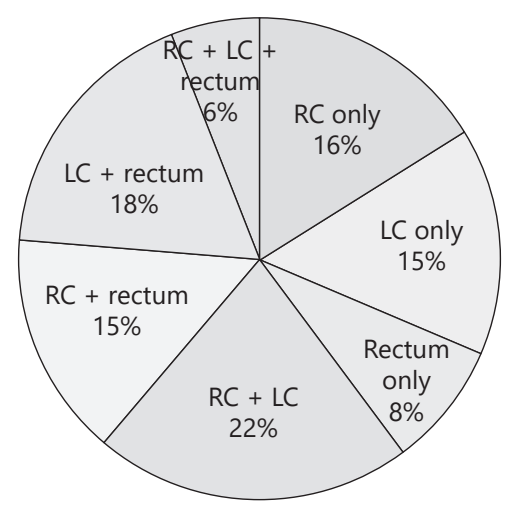

Fig. 4. Distribution of the multiple CRCs. CRCs, colorectal cancers; RC, right-side colon; LC, left-side colon.

The influence of MPMs on CRC prognoses was not fully investigated until now. In particular, there have been few reports on the prognoses of patients with EMs. In the present study, we investigated the prognostic impact in both SCRCs and SEMs. The multivariate analysis showed that the presence of SEMs was strongly associated with a poor prognosis. Notably, the presence of SEMs (HR 2.21) was the strongest poor prognostic factor, exceeding age (HR 2.02), primary T factor (HR 1.89), and $\mathrm{N}$ factor (HR 2.03). We also performed a multivariate analysis using stomach, lung, prostate, and breast cancers, which were the most common types of EM, as independent variables (online suppl. Table). The results suggested that stomach and lung cancers, which generally have worse prognoses than CRC, are associated with a poor prognosis, while breast and prostate cancers, which have better prognoses, may not be associated with a poor prognosis for CRC. Since CRC has a relatively good prognosis, and long-term survival can be achieved with appropriate treatment, it is important not only to consider the disease stage of primary CRC but also preoperative screening and postoperative surveillance of EMs, which have a worse prognosis than $\mathrm{CRC}$, and to control them. In most previous reports that described the prognoses of patients with CRC, the SEMs factor was not recognized and just regarded as another cause of death in the evaluation of OS and RFS [14]. However, the finding that SEMs have a greater prognostic impact than other factors is extremely important; thus, clinicians should keep in mind that pa-

tients with CRC may die of cancers other than CRC when deciding treatment options and surveying. Furthermore, when conducting research to evaluate CRC prognoses, we must design studies that take into account the presence of SEMs- and EMs-related death. By contrast, SCRCs did not affect prognoses in the present study, which is consistent with many previous reports $[21,22]$. Several other studies reported that the presence of SCRCs was a poor prognostic factor. Nosho et al. [10] and Oya et al. [11] showed that the patients with SCRCs had poorer prognoses than those without SCRCs in the univariate analysis; conversely, there was no significant difference in the multivariate analysis adjusted for disease stage and curability. Moreover, none of these previous reports considered the presence of SEMs in the multivariate analyses. Patients with SCRCs are more likely to develop distant or lymph node metastasis concomitantly than those without SCRCs, which could be associated with the poorer prognoses. However, as shown by our results and many previous reports $[11,12$, $22]$, the prognoses do not appear to differ when comparing patients who receive curative resection and adjusting for disease stage.

Several hereditary diseases are known to lead to CRC. For example, Lynch syndrome is known to have mutations in the mismatch repair gene, which leads to a risk of colorectal, endometrial, stomach, ovarian, biliary tract, ureteral, and small intestinal cancers [23]. As shown in Table 5, stomach cancer was the most common type in the present study, followed by lung, breast, prostate, and uterine cancers. This population's characteristics differ from Lynch syndrome in that there was a large proportion of breast and prostate cancer. According to a report from the Japanese Ministry of Health, Labour, and Welfare, except for CRC, stomach cancer has the highest incidence rate among malignant diseases in Japan, with a rate of 136.1 per 100,000 population, followed by lung cancer (115.4 per 100,000$)$ and prostate cancer $(96.0$ per 100,000$)$. Breast cancer $(93.7$ per 100,000$)$ is the most common in women, followed by stomach cancer $(58.2$ per 100,000$)$ and lung cancer $(48.9$ per 100,000$)$ [5]. Our results are similar to these findings, but there was a notably high proportion of stomach cancer in our population. In men, in particular, there were nearly twice as many cases of stomach cancer than lung cancer and prostate cancer. Kato et al. [7] reported a large proportion of stomach cancer as MPMs in patients with CRC. They noted that the incidence of stomach cancer with CRC was significantly higher compared to the healthy population collected 
from mass screenings $(0.53$ vs. $0.03 \%, p<0.001$, OR 18.8) [7]. We obtained a result supporting that of Kato et al. [7] in a larger, multi-institute cohort. Although there are some limitations as shown below, MPMs with CRC were characterized by a higher proportion of stomach cancers, which differs from the general population and Lynch syndrome. Therefore, the stomach should be carefully surveyed, such as via GS in preoperative screening, in patients with CRC.

The reported distribution of multiple CRCs greatly varies $[11,21,24]$. In the present study, CRCs were evenly distributed throughout the large intestine. In addition, there was a relatively small population with multiple CRCs only in the rectum. Therefore, it is important to examine the entire colon and rectum for preoperative screening and postoperative surveillance.

There are several limitations in this study. First, it was retrospective. The other limitation was based on the selection biases. For example, many patients with CRC undergo chest CT before surgery. GS is also often performed in Japan. Conversely, serum PSA testing is rarely performed. These screening tests generally increase the apparent incidence of cancer through early detection. The resulting selection bias may affect the frequency and distribution of MPMs. Besides, because exhaustive surveillance for all types of cancers was not routinely performed during the postoperative followup period, information about EMs during this period might be insufficient. Another limitation was that disease stage of the second CRC is not fully described in our data. We must consider that there might be contained pathological in situ cancers as secondary CRCs in multiple patients with CRC. Furthermore, the lack of information, such as genetic background (i.e., p53, MSI, and RAS), family history, social history, comorbidities, and $H$. pylori infection, is also a major limitation. Information about the onset and disease stage of metachronous MPMs was missing from our data. The proportions of MEM and SEM among EM-related deaths were also unknown. Genetic information containing MSI was inadequate because the study population included patients from a relatively older era when hereditary CRC was not well recognized. It should be noted that SCRC might be more prone to being MSI high due to mutations in the MMR gene, even in sporadic CRCs. MSI-high CRC is generally considered to have a better prognosis than MSI-stable CRC, except in the case of recurrent or metastatic CRC [25], which may consequently mask the effects of secondary CRC. In addition, the number of excluded hereditary CRCs was only 30 in 22,379 patients. The incidence of hereditary CRC has been reported to be up to $5-7 \%$ among all CRCs [26]; our results were much lower than this. The diagnoses of hereditary diseases generally require detailed family history and prior medical history in addition to genetic information; however, this information might not be specified in the medical record, where it might be listed as underdiagnosis. Further studies focusing on the above issues are required in the future.

In conclusion, the presence of SEMs was a strong poor prognostic factor for patients with stage I-III CRC. Patients with SCRCs were more likely to have SEMs. Because stomach and lung cancers were common as SEMs, it is recommended that they are surveyed when treating patients with CRC, particularly in those with SCRCs.

\section{Acknowledgment}

This study is based on data from 22 hospitals that are members of the Japanese Study Group for Postoperative Follow-up of CRC: Ichiro Takemasa (Sapporo Medical University); Kenichi Hakamada (Hirosaki University); Hitoshi Kameyama (Niigata University); Yasukimi Takii (Niigata Cancer Center Hospital); Hideki Ueno (National Defense Medical College); Heita Ozawa (Tochigi Cancer Center); Soichiro Ishihara (the University of Tokyo); Keiichi Takahashi (Tokyo Metropolitan Cancer and Infectious Diseases Center Komagome Hospital); Yukihide Kanemitsu (National Cancer Center Hospital); Michio Itabashi (Tokyo Women's Medical University); Tomomichi Kiyomatsu (National Center for Global Health and Medicine); Yusuke Kinugasa (Tokyo Medical and Dental University); Takeshi Okabayashi (Keio University); Yojiro Hashiguchi (Teikyo University); Tadahiko Masaki (Kyorin University); Masahiko Watanabe (Kitasato University); Akio Shiomi (Shizuoka Cancer Center); Kouichi Hanai (Fujita Health University); Koji Komori (Aichi Cancer Center Hospital); Yoshiharu Sakai (Kyoto University); Masayuki Ohue (Osaka Medical Center for Cancer and Cardiovascular Diseases); Shingo Noura (Osaka Rosai Hospital); Naohiro Tomita (Hyogo College of Medicine); and Yoshito Akagi (Kurume University).

\section{Statement of Ethics}

The Ethics Committee of the University of Tokyo (No. 2018028NI-[1]) and local ethics committees in each participant institute approved the study protocol. The study was conducted in accordance with the Declaration of Helsinki. Informed consent was replaced by the obligation of information to the participants and the right of participants to opt out due to the study's retrospective nature. 


\section{Conflict of Interest Statement}

The authors have no conflicts of interest to declare.

\section{Funding Sources}

The authors did not receive any funding.

\section{Author Contributions}

Kentaro Ochiai designed the study and wrote the initial draft of the manuscript. Kazushige Kawai contributed to analysis and interpretation of the data and assisted in the preparation of the manuscript. All other authors have contributed to data collection and interpretation and have critically reviewed the manuscript. All authors approved the final version of the manuscript and agree to be accountable for all aspects of the work in ensuring that questions related to the accuracy or integrity of any part of the work are appropriately investigated and resolved.

\section{References}

1 Ferlay J, Soerjomataram I, Dikshit R, Eser S, Mathers C, Rebelo M, et al. Cancer incidence and mortality worldwide: sources, methods and major patterns in GLOBOCAN 2012. Int J Cancer. 2015;136(5):E359-86.

2 Levi F, Randimbison L, Blanc-Moya R, Maspoli-Conconi M, Rosato V, Bosetti C, et al. High constant incidence of second primary colorectal cancer. Int J Cancer. 2013;132(7) 1679-82.

3 Yang L, Xiong Z, Xie QK, He W, Liu S, Kong $\mathrm{P}$, et al. Second primary colorectal cancer after the initial primary colorectal cancer. BMC Cancer. 2018;18(1):931.

4 Evans HS, Møller H, Robinson D, Lewis CM, Bell CM, Hodgson SV. The risk of subsequent primary cancers after colorectal cancer in southeast England. Gut. 2002 May;50(5):64752.

5 Hori M, Matsuda T, Shibata A, Katanoda K, Sobue T, Nishimoto $\mathrm{H}$. Cancer incidence and incidence rates in Japan in 2009: a study of 32 population-based cancer registries for the monitoring of cancer incidence in Japan (MCIJ) project. Jpn J Clin Oncol. 2015 Sep; 45(9):884-91.

6 Tsukuma H, Fujimoto I, Hanai A, Hiyama T, Kitagawa T, Kinoshita N. Incidence of second primary cancers in osaka residents, Japan, with special reference to cumulative and relative risks. Jpn J Cancer Res. 1994;85(4):33945.

7 Kato T, Suzuki K, Muto Y, Sasaki J, Tsujinaka S, Kawamura YJ, et al. Multiple primary malignancies involving primary sporadic colorectal cancer in Japan: incidence of gastric cancer with colorectal cancer patients may be higher than previously recognized. World J Surg Oncol. 2015;13(1):23.

8 Kawai K, Ishihara S, Nozawa H, Hata K, Kiyomatsu T, Tanaka T, et al. Survival impact of extracolorectal malignancies in colorectal cancer patients. Digestion. 2016;94(2):92-9.
9 Bekdash B, Harris S, Broughton CI, Caffarey SM, Marks CG. Outcome after multiple colorectal tumours. Br J Surg. 1997;84(10): $1442-4$.

10 Nosho K, Kure S, Irahara N, Shima K, Baba Y, Spiegelman D, et al. A prospective cohort study shows unique epigenetic, genetic, and prognostic features of synchronous colorectal cancers. Gastroenterology. 2009; 137(5): 1609-3.

11 Oya M, Takahashi S, Okuyama T, Yamaguchi M, Ueda Y. Synchronous colorectal carcinoma: clinico-pathological features and prognosis. Jpn J Clin Oncol. 2003;33(1):38-43.

12 Arakawa K, Hata K, Nozawa H, Kawai K, Tanaka T, Nishikawa T, et al. Prognostic significance and clinicopathological features of synchronous colorectal cancer. Anticancer Res. 2018;38(10):5889-95.

13 Yang J, Peng JY, Chen W. Synchronous colorectal cancers: a review of clinical features, diagnosis, treatment, and prognosis. Dig Surg. 2011;28(5-6):379-85.

14 Punt CJA, Buyse M, Köhne C-H, Hohenberger P, Labianca R, Schmoll HJ, et al. Endpoints in adjuvant treatment trials: a systematic review of the literature in colon cancer and proposed definitions for future trials. J Natl Cancer Ins. 2007;99(13):998-1003.

15 Bellera CA, Pulido M, Gourgou S, Collette L, Doussau A, Kramar A, et al. Protocol of the definition for the assessment of time-to-event endpoints in CANcer trials (DATECAN) project: formal consensus method for the development of guidelines for standardised time-to-event endpoints' definitions in cancer clinical trials. Eur J Cancer. 2013;49(4): 769-81.

16 Japanese Society for Cancer of the Colon and Rectum. General rules for clinical and pathological studies on cancer of the colon, rectum and anus. 7th ed. Tokyo: Kanehara \& Co., Ltd.; 2009.
17 Watanabe T, Itabashi M, Shimada Y, Tanaka S, Ito Y, Ajioka Y, et al. Japanese society for cancer of the colon and rectum (JSCCR) guidelines 2010 for the treatment of colorectal cancer. Int J Clin Oncol. 2012;17(1):1-29.

18 Warren S, Gates O. Multiple primary malignant tumors. A survey of the literature and a statistical study. Am J Cancer. 1932;16:1358414.

19 Watson P, Lynch HT. Extracolonic cancer in hereditary nonpolyposis colorectal cancer. Cancer. 1993 Feb 1;71(3):677-85.

20 Research WCRFAIfC. Diet, nutrition, physical activity and cancer: a global perspective continuous update project expert report; 2018.

21 Latournerie M, Jooste V, Cottet V, Lepage C, Faivre J, Bouvier AM. Epidemiology and prognosis of synchronous colorectal cancers. Br J Surg. 2008;95(12):1528-33.

22 Enker WE, Dragacevic S. Multiple carcinomas of the large bowel: a natural experiment in etiology and pathogenesis. Ann Surg. 1978 Jan; 187(1):8-11.

23 Lynch HT, Smyrk TC, Watson P, Lanspa SJ, Lynch JF, Lynch PM, et al. Genetics, natural history, tumor spectrum, and pathology of hereditary nonpolyposis colorectal cancer: an updated review. Gastroenterology. 1993; 104(5):1535-49.

24 Passman MA, Pommier RF, Vetto JT. Synchronous colon primaries have the same prognosis as solitary colon cancers. Dis Colon Rectum. 1996 Mar;39(3):329-34.

25 Boland CR, Thibodeau SN, Hamilton SR, Sidransky D, Eshleman JR, Burt RW, et al. A national cancer institute workshop on microsatellite instability for cancer detection and familial predisposition: development of international criteria for the determination of microsatellite instability in colorectal cancer. Cancer Res. 1998 Nov 15;58(22):5248-57.

26 Dekker E, Tanis PJ, Vleugels JLA, Kasi PM, Wallace MB. Colorectal cancer. Lancet. 2019 Oct 19;394(10207):1467-80. 